\title{
Osmotic Demyelination Syndrome after Primary Hypoadrenocorticism Crisis Management
}

\author{
Álan Gomes Pöppl $\oplus_{\odot}^{1,2}$, Érico Haas Pires $\oplus^{2}$, Claudia Ruga Barbieri ${ }^{3}$ \& Lucas Marques Colomé ${ }^{4}$
}

\begin{abstract}
Background: Primary hypoadrenocorticism is a rare condition resulting from immune-mediated destruction of the adrenal cortices. It can also occur due to necrosis, neoplasms, infarctions, and granulomas. The clinical and laboratory changes are due to deficient secretion of glucocorticoids and mineralocorticoids, which leads to electrolyte disorders associated with hyponatremia and hyperkalemia. These disorders can cause hypotension, hypovolemia and shock, putting a patient's life at risk if inadequate hydroelectrolytic supplementation and hormone replacement is provided. Nevertheless, rapid sodium chloride supplementation is contraindicated due to the risk of central pontine myelinolysis induction. The present study aims to describe a thalamic osmotic demyelination syndrome after management of a primary hypoadrenocorticism crisis in a 2-year-old, female West White Highland Terrier.

Case: The patient had a presumptive diagnosis of hypoadrenocorticism already receiving oral prednisolone and gastrointestinal protectants in the last 2 days. After prednisolone dose reduction the dog presented a severe primary hypoadrenocorticism crisis treated with intravenous sodium chloride $0.9 \%$ solution along with supportive therapy. Four days after being discharged from the hospital, the patient showed severe neurological impairment and went back to the clinic where a neurological examination revealed mental depression, drowsiness, ambulatory tetraparesis and proprioceptive deficit of the 4 limbs, postural deficits, and cranial nerves with decreased response. Due to these clinical signs, a magnetic resonance imaging was performed. It showed 2 intra-axial circular lesions, symmetrically distributed in both thalamus sides, with approximately $0.8 \mathrm{~cm}$ in diameter each without any other anatomical changes on magnetic resonance imaging. The images were compatible with metabolic lesions, suggesting demyelination. Furthermore, liquor analysis did not show relevant abnormalities, except for a slight increase in density and $\mathrm{pH}$ at the upper limit of the reference range. After treatment, the patient had a good neurological evolution secondary to standard primary hypoadrenocorticism treatment, without sequelae. Discussion: In the present case report, primary hypoadrenocorticism gastrointestinal signs seemed to be triggered by a food indiscretion episode, not responsive to the symptomatic therapies employed. The patient's breed and age (young West White Highland Terrier bitch) is in accordance with the demographic profile of patients affected by the disease, where young females are frequently more affected. Regarding the probable thalamic osmotic demyelination syndrome documented in this case, is important to notice that myelinolysis or demyelination is an exceedingly rare noninflammatory neurological disorder, initially called central pontine myelinolysis, which can occur after rapid correction of hyponatremia. It has already been observed in dogs after correction of hyponatremia of different origins, including hypoadrenocorticism and parasitic gastrointestinal disorders. Currently, the terms "osmotic myelinolysis" or "osmotic demyelination syndrome" are considered more suitable when compared to the term "central pontine myelinolysis" since it has been demonstrated in dogs and humans the occurrence of demyelination secondary to the rapid correction of hyponatremia in distinct regions of the central nervous system including pons, basal nuclei, striatum, thalamus, cortex, hypoppocampus, and cerebelum. The present case report emphasizes the difficulties for hormonal confirmation of primary hypoadrenocorticism in a patient already on corticosteroid treatment, as well as proposes that the current term osmotic demyelination syndrome replace the term "central pontine myelinolysis" in veterinary literature related to the management of hypoadrenocorticism crisis.
\end{abstract}

Keywords: Addison Syndrome, hyponatremia, osmotic myelinolysis, magnetic resonance imaging. 


\section{INTRODUCTION}

Primary hypoadrenocorticism (PHA) is a rare condition resulting from immune-mediated destruction of the adrenal cortices $[11,19]$. It can also occur due to necrosis, neoplasms, infarctions, and granulomas [11]. The clinical and laboratory changes are due to deficient secretion of glucocorticoids and mineralocorticoids, leading to electrolyte disorders associated with hyponatremia and hyperkalemia [21]. However, in cases known as atypical hypoadrenocorticism, patients seem able to compensate for mineralocorticoid function without developing electrolyte disorders, even in aldosterone deficiency [22]. In contrast, patients with secondary hypoadrenocorticism resulting from hypothalamic-pituitary lesions and deficiency of the adrenocorticotrophic hormone (ACTH) do not present deficit in mineralocorticoid secretion, only in glucocorticoids [21]. From a clinical approach, it is not possible to distinguish different forms of the disease without adequate hormonal tests [6].

In the presence of hyponatremia and depending on the severity of the mineralocorticoid deficiency, hypotension, hypovolemia and shock may happen, putting the patient's life at risk if inadequate hydroelectrolytic supplementation and hormone replacement is provided [21]. Rapid sodium supplementation is contraindicated due to the risk of central pontine myelinolysis induction [16]. In human medicine, myelinolysis is described in the pons and other sites of the central nervous system $[14,15,18]$. Therefore, the term osmotic demyelination syndrome (ODS) is being currently adopted to describe this neurologic condition [14]. The aim of this report is to describe a probable thalamic ODS case after PHA crisis management in a dog.

\section{CASE}

A 2-year-old West White Highland Terrier intact bitch, weighing $4.8 \mathrm{~kg}$, was admitted at the Endocrinology and Metabology Service at the Veterinary Medical Teaching Hospital (HCV) of the Federal University of Rio Grande do Sul (UFRGS), due to a previous presumptive diagnosis of hypoadrenocorticism. Twelve days earlier, after an episode of dietary indiscretion, the patient started to present emesis, hyporexia, and prostration; with no changes in stools. The patient was taken to a private clinic where a symptomatic therapy for gastroenteritis was performed. However, there was no improvement of the initial presentation, with progression to generalized weakness, tremors, and staggering walk. The owners took the patient to another clinic where a presumptive diagnosis of hypoadrenocorticism was given and therapy with prednisolone ${ }^{1}$ [1 $\mathrm{mg} / \mathrm{kg} \mathrm{q} 12 \mathrm{~h}$ ], in association with omeprazole ${ }^{2}[1 \mathrm{mg} / \mathrm{kg} \mathrm{q} 12 \mathrm{~h}]$ and ranitidine hydrochloride ${ }^{3}$ [2 $\mathrm{mg} / \mathrm{kg} \mathrm{q} 12 \mathrm{~h}$ ] was started. The owners were administering homemade serum (water, salt, and sugar) for rehydration. However, they considered the clinical response unsatisfactory due to the persistence of signs such as hyporexia, emesis, aquesia, and weakness. Despite these problems, the owners reported normuria and normodipsy.

At clinical examination, the patient showed low body condition score (4/1-9), muscle mass loss (2/0-3), rectal temperature of $37.6^{\circ} \mathrm{C}$, normal colored oral mucosa and capillary refill time of less than $2 \mathrm{~s}$ despite discreet dehydration $(<5 \%)$. The animal was depressed and showing no signs of abdominal pain or discomfort on palpation. Its heart rate was $102 \mathrm{bpm}$, with a steady pulse and average systolic blood pressure of $123 \mathrm{mmHg}$. A set of blood tests was performed (Table 1, time 0), including an ACTH stimulation test ${ }^{4}$ [tetracosactide - $5 \mu \mathrm{g} / \mathrm{kg}$, IV] which revealed a baseline cortisol value of $4.04 \mathrm{mcg} / \mathrm{dL}$ (reference range: $0.5-6 \mu \mathrm{g} / \mathrm{dL}$ ) and a post-ACTH cortisol of $2.2 \mu \mathrm{g} / \mathrm{dL}$ (reference range: 6-17 $\mu \mathrm{g} / \mathrm{dL}$ ). Cortisol measurements were determined by radioimmunoassay ${ }^{5}$ (Immuchem). However, the patient was receiving prednisolone for the past $48 \mathrm{~h}$ and had received a dose of $1 \mathrm{mg} / \mathrm{kg}$ at home, $2 \mathrm{~h}$ prior to the test. An abdominal ultrasound scanning using either a linear 12-7.5 MHz and a curved 8-5.5 MHz electric array transducer and a My Lab TM 40 ultrasound system ${ }^{6}$ showed hypoechoic and homogeneous adrenals, with the left adrenal measuring $1.2 \times 0.34 \mathrm{~cm}$ in the caudal pole and the right adrenal measuring $1.56 \times 0.4 \mathrm{~cm}$ in the caudal pole. Adrenal measurements were made in the sagittal plane. The spleen and liver showed a slight reduction in their dimensions, both with hypoechoic parenchyma. It also showed a gastric wall mucosa thickening $(0.6 \mathrm{~cm})$ as well as colonic mucosa thickening $(0.5 \mathrm{~cm})$, with no other changes in the abdominal cavity.

The owners choose not to admit the animal at the hospital, so home therapy was indicated to reduce gradually the oral prednisolone intake, initially to $0.5 \mathrm{mg} / \mathrm{kg} \mathrm{q} 12 \mathrm{~h}$ on the first day, followed by $0.25 \mathrm{mg} / \mathrm{kg} \mathrm{q} 12 \mathrm{~h}$ on the second day, in association 
with fludrocortisone ${ }^{7}[0.01 \mathrm{mg} / \mathrm{kg} \mathrm{q} 12 \mathrm{~h}]$. Approximately $36 \mathrm{~h}$ after the appointment, while receiving $0.5 \mathrm{mg} / \mathrm{kg}$ of prednisolone $\mathrm{q} 12 \mathrm{~h}$, the patient had severe hemorrhagic gastroenteritis, and was admitted to the private clinic in which presumptive PHA diagnosis was made. Fluid therapy with sodium chloride $0.9 \%$ was performed, as well as the administration of gastroprotectants and fludrocortisone, in association with dexamethasone ${ }^{8}$ [0.5 mg/kg q12 h intravenously]. New blood tests were performed after $12 \mathrm{~h}$ of hospitalization (Table 1, day 2) and the patient remained at the clinic for 3 days until stabilizing and resuming voluntary feeding. She was then discharged with recommendation of maintenance of prednisolone $[0.25 \mathrm{mg} / \mathrm{kg} \mathrm{q} 12 \mathrm{~h}$ followed by a gradual reduction to $0.1 \mathrm{mg} / \mathrm{kg} \mathrm{q} 24 \mathrm{~h}]$, in association with fludrocortisone [0.01 mg/kg, q $12 \mathrm{~h}]$.

After 4 days at home, keeping normal appetite and in the absence of gastrointestinal signs, the owner brought the dog back to the hospital due to severe uncoordinated movements. This led to a new hospitalization, and another set of hematological and serum biochemistry tests, which were performed about $12 \mathrm{~h}$ after hospitalization (Table 1, day 7). At clinical examination, the patient was adequately hydrated, with normal colored mucosa, capillary refill time of less than $2 \mathrm{~s}$ and mean arterial pressure of $160 \mathrm{mmHg}$. Neurological examination revealed mental depression/ drowsiness, ambulatory tetraparesis, proprioceptive deficit of the four limbs, postural deficits, and cranial nerves with decreased response (Figure 1A).

Due to a clinical suspicion of encephalitis, magnetic resonance imaging (MRI) of the skull and cerebrospinal fluid analysis (CSF) of the patient were requested. The MRI exam was performed under inhalation anesthesia using T1 and T2 weighted image scans, as well as Flair and Diffusion weighted imaging, which were all obtained in the sagittal, transverse and dorsal projections, in addition to T1 weighted images performed after administration of intravenous contrast. The MRI showed 2 intra-axial circular lesions, symmetrically distributed, one in the left thalamus and the other in the right thalamus, with approximately $0.8 \mathrm{~cm}$ in diameter each, with hypersignal in the weighted images in T2 and Flair, normal signal in T1, moderate Diffusion restriction and absence of contrast impregnation (Figure 2). The images were considered compatible with metabolic lesions, suggesting demyelination. The CSF analysis showed no changes, except for a slight increase in density and $\mathrm{pH}$ at the upper limit of the reference range (Table 2).

The patient was kept under monitoring during hospitalization for 5 days, in therapy with oral prednisolone $[0.25 \mathrm{mg} / \mathrm{kg} \mathrm{q} 12 \mathrm{~h}]$ and fludrocortisone $[0.01 \mathrm{mg} / \mathrm{kg} \mathrm{q} 12 \mathrm{~h}]$. The patient showed a positive evolution of the neurological condition during the period and after discharge from the clinic, therapy with fludrocortisone was replaced by deoxycorticosterone pivalate $^{9}$ [2 $\mathrm{mg} / \mathrm{kg}$ subcutaneously every 30 days]. Concomitantly, the prednisolone dose was gradually reduced [over the next 2 weeks to $0.15 \mathrm{mg} / \mathrm{kg} \mathrm{q} 48 \mathrm{~h}$ ] due to owners' complaints of polyphagia, polyuria, and polydipsia. In subsequent clinical visits, the patient remained clinically stable, with controlled appetite and in absence of other gastrointestinal manifestations. Regarding neurological changes, the patient showed complete clinical recovery and did not present any ambulatory deficit nor neurological alterations during

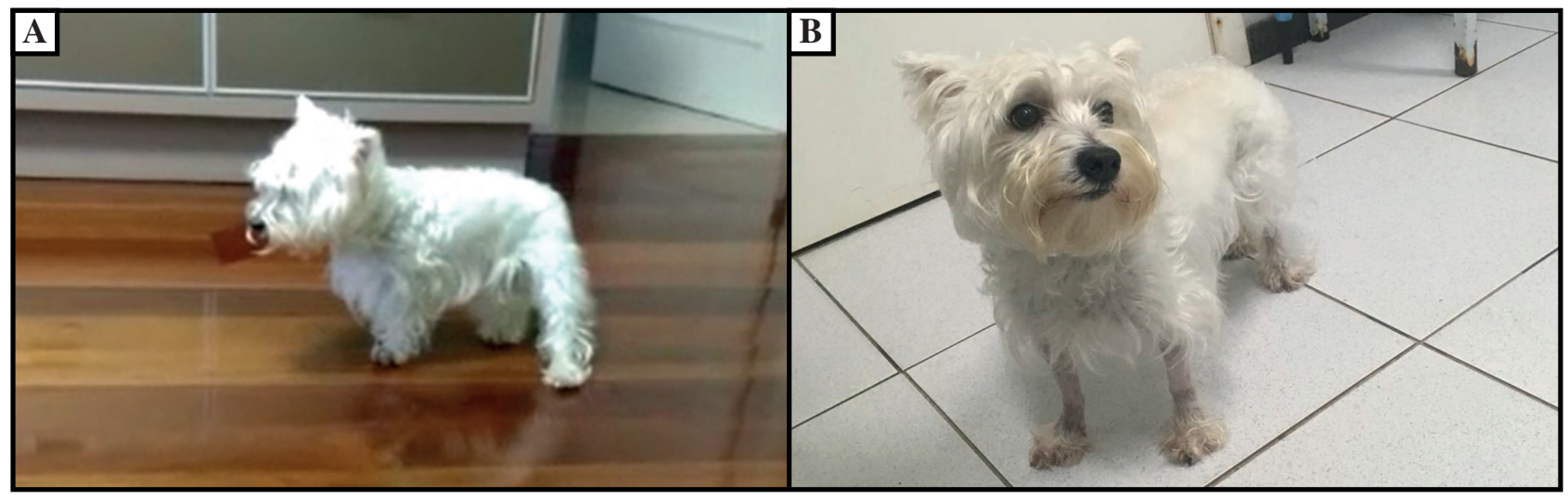

Figure 1. A- Subacute presentation of mental dullness, ataxia, and difficulty to stand a few days after hypoadrenocorticism crisis management. B- Complete resolution of neurological signs a few weeks after subacute presentation. A video of the neurological features observed at home and the clinics may be seen in the following link: https://youtu.be/0T8FolfpnpY 
A.G. Pöppl, E.H. Pires, C.R. Barbieri \& L.M. Colomé. 2021. Osmotic Demyelination Syndrome after Primary Hypoadrenocorticism Crisis Management. Acta Scientiae Veterinariae. 49(Suppl 1): 682.

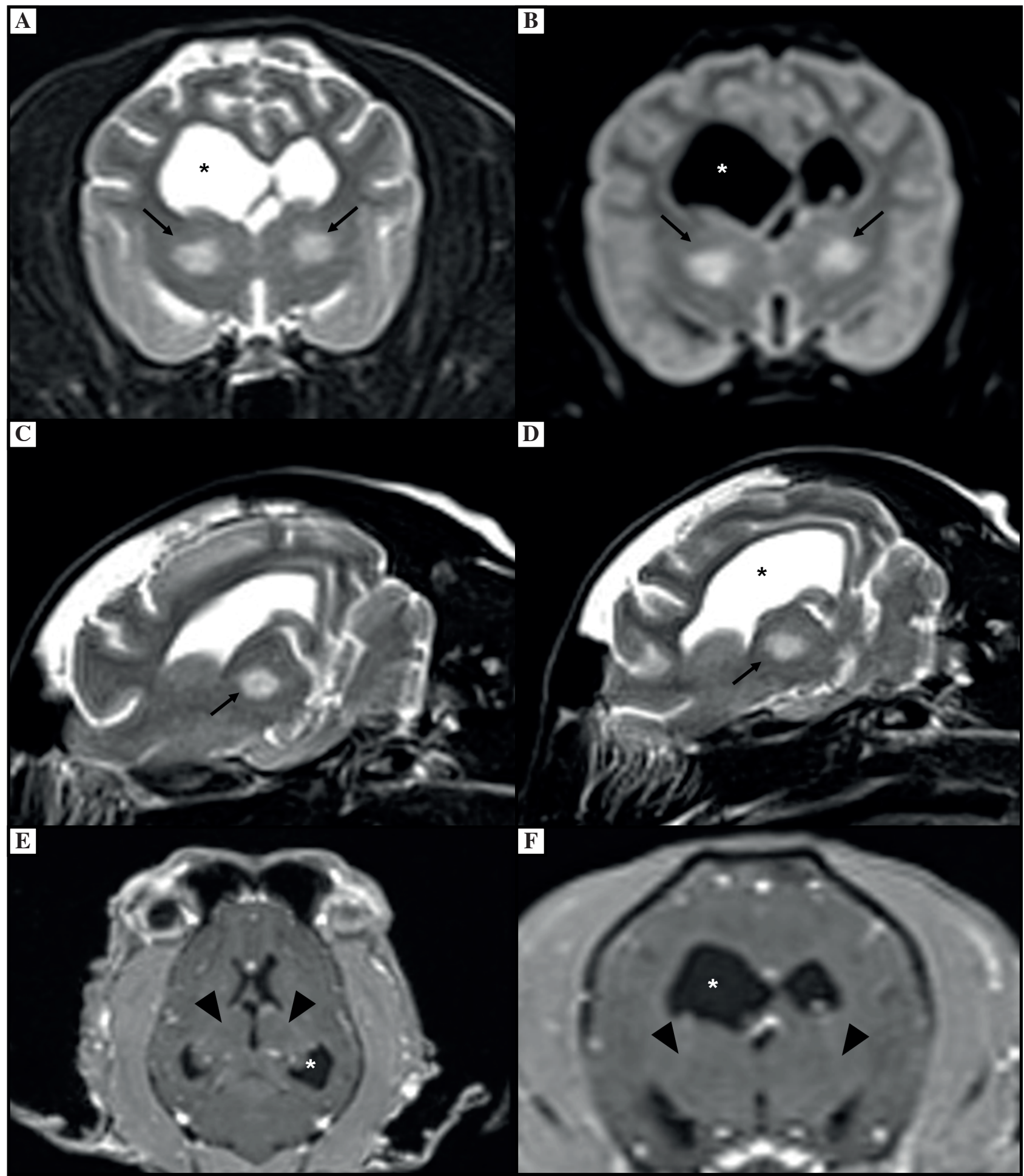

Figure 2. Transverse magnetic resonance images (1.5 tesla) showing hypersignal (arrow) in the T2-weighted (A) and Flair (B) images bilaterally in the thalamus region; and sagittal section showing bilateral hypersignal (arrow) in the thalamus region in the T2-weighted image (C, D). The lesions are about $0.8 \mathrm{~cm}$ in diameter each, with an asymmetry of lateral ventricles $\left(^{*}\right)$ and preserved midline. After administration of intravenous contrast, T1-weighted images show no contrast impregnation (arrowhead) in axial (E), and transversal (F) sections.

examination (Figure 1B). An increase in body weight to $6.85 \mathrm{~kg}$ was also reported, reaching a body condition score of 6/1-9, with muscle mass gain (muscle mass score 3/0-3). The patient also kept stable systolic blood pressure, with a mean of $135 \mathrm{mmHg}$, and general tests showed no evidence of hypoadrenocorticism, iatrogenic hypercortisolism and/or hyperaldosteronism, which could happen due to the monthly use of deoxycorticosterone (Table 1, day 30) that could occur if the PHA diagnosis was wrong. 
Table 1. Hematological and serum biochemistry parameters of the patient throughout the follow-up period.

\begin{tabular}{|c|c|c|c|c|c|c|}
\hline Parameter & Day 0 & Day 2 & Day 7 & Day 30 & Day 235 & Reference \\
\hline Erythrocytes $\times 10^{6} / \mathrm{mm}^{3}$ & 6.96 & 6.11 & 4.22 & 6.65 & 6.81 & $5.5-8.5$ \\
\hline Hematocrit (\%) & 48 & 40 & 29 & 47 & 45 & $37-55$ \\
\hline Hemoglobin (g/dL) & 16.4 & 12.5 & 8.6 & 16.4 & 16.1 & $12-18$ \\
\hline Leukocytes $\times 10^{3} / \mathrm{mm}^{3}$ & 23 & 21.9 & 25.2 & 10.2 & 13.9 & $6-17$ \\
\hline Segmented $\times 10^{3} / \mathrm{mm}^{3}$ & 17.1 & 19.7 & 23.4 & 6.94 & 9.86 & $3-11.4$ \\
\hline Eosinophils $\times 10^{3} / \mathrm{mm}^{3}$ & 0 & 0.22 & 0 & 0.2 & 0.28 & $0.1-0.75$ \\
\hline Limphocytes $\times 10^{3} / \mathrm{mm}^{3}$ & 3.45 & 1.32 & 1.26 & 2.75 & 3.06 & $1-4.8$ \\
\hline Monocytes x $10^{3} / \mathrm{mm}^{3}$ & 1.84 & 0.66 & 0.5 & 0.3 & 0.69 & $0.15-1.35$ \\
\hline Platelets $\times 10^{3} / \mathrm{mm}^{3}$ & 162 & 220 & 170 & 294 & 284 & $200-500$ \\
\hline Albumin (g/dL) & 3.26 & 2.76 & 3.25 & 3.13 & 3.23 & $2.3-3.8$ \\
\hline $\operatorname{ALP}^{\mathrm{a}}(\mathrm{U} / \mathrm{L})$ & 55.1 & 40.5 & 70.9 & 52.6 & 76.1 & $<152$ \\
\hline $\operatorname{ALT}^{\mathrm{b}}(\mathrm{U} / \mathrm{L})$ & 52.3 & 32.1 & 31.2 & 34.4 & 46.4 & $<102$ \\
\hline Chlorine (mg/dL) & 83 & - & - & 109 & 120 & $105-120$ \\
\hline Cholesterol (mg/dL) & 84.4 & - & - & 146.3 & 155 & $135-270$ \\
\hline Creatinin (mg/dL) & 0.5 & 0.69 & 0.52 & 1.1 & 0.78 & $0.5-1.5$ \\
\hline Phosphorus (mg/dL) & 3.46 & - & - & 5.57 & 4.39 & $2.6-6.8$ \\
\hline Glucose (mg/dL) & 118 & - & 140 & 97 & 77.6 & $60-110$ \\
\hline Potassium (mg/dL) & 4.8 & 5.1 & 4.3 & 4.6 & 4.04 & $3.9-5.6$ \\
\hline Sodium (mg/dL) & 141 & 130.6 & 148.9 & 144.3 & 143.2 & $141-154$ \\
\hline Na:Kc Ratio & $29: 1$ & $25: 1$ & $34: 1$ & $31: 1$ & $35: 1$ & $>30: 1$ \\
\hline Total calcium (mg/dL) & 9.3 & - & - & 10.6 & 8.9 & $8-12$ \\
\hline $\operatorname{TPP}^{d}(\mathrm{~g} / \mathrm{dL})$ & 5.2 & 5.5 & 6.6 & 6.0 & 6.2 & $5.5-8.0$ \\
\hline Triglycerides (mg/dL) & 25.3 & - & - & 119 & 40.5 & $32-138$ \\
\hline Urea (mg/dL) & 34.2 & 24.7 & 27.2 & 42.7 & 20.8 & $10-60$ \\
\hline
\end{tabular}

alkaline phosphatase; balanine aminotransferase ${ }^{\mathrm{c}}$ sodium: potassium; ' ${ }^{\mathrm{d}}$ total plasmatic protein.

In order to confirm the diagnosis of hypoadrenocorticism, an endogenous ACTH (eACTH) measurement was requested and determined with samples collected in pre-cooled EDTA tubes, which were immediately centrifuged. The resultant plasma was stored at $-80^{\circ} \mathrm{C}$ and later transported on dry ice for electrochemiluminescence analysis (Immulite 1000) ${ }^{10}$. This test was performed 6 weeks after the patient reached the prednisolone dose of $0.15 \mathrm{mg} / \mathrm{kg} \mathrm{q} 48 \mathrm{~h}$, being collected $48 \mathrm{~h}$ after the last oral administration of prednisolone. The eACTH result was $136 \mathrm{pg} / \mathrm{mL}$ (reference range: $10-25 \mathrm{pg} / \mathrm{mL}$ ) which was considered compatible with primary hypoadrenocorticism. Eight months after the reported events, the patient remained clinically stable with the therapy described above, with absence of hypoadrenocorticism, clinical signs or neurological changes, and keeping all laboratory tests within the normal range (Table 1, day 235).

\section{DISCUSSION}

The main characteristics of this case report were: 1) the difficulties in confirming the PHA diagnosis in a patient already in treatment with prednisolone, and 2) occurrence of probable osmotic demyelination after therapy to manage a hypoadrenocorticism crisis. Frequently, patients with hypoadrenocorticism develop clinical signs after exposure to an episode of stress that works as a trigger for the onset of the clinical manifestations of the disease [21]. In the present case report, apparently, PHA gastrointestinal signs were 
Table 2. Patient's cerebrospinal fluid (CSF) analysis (physical, biochemical, and cytological) results. The sample was obtained under general anesthesia from the cisterna magna during the magnetic resonance imaging.

\begin{tabular}{ccc}
\hline Parameter & Result & Reference \\
\hline Volume $(\mathrm{mL})$ & 1.00 & Clear \\
Color & Clear & Clear or translucent \\
Aspect & Translucent & 7.00 to 9.00 \\
pH & 9.00 & $1,003-1,006$ \\
Specific Gravity & 1,008 & 0 a 5 \\
Nucleated cells $\left(/ \mathrm{mm}^{3}\right)$ & 1.00 & \\
Macrophages $(\%)$ & 40 & up to 30 \\
Segmented $(\%)$ & 60 & $40-80$ \\
Erythrocytes $\left(/ \mathrm{mm}^{3}\right)$ & 2.00 & $25-30$ \\
Glucose $(\mathrm{mg} / \mathrm{dL})$ & 74 & No microorganism \\
Proteins $(\mathrm{mg} / \mathrm{dL})$ & 28 & Negative \\
Microorganisms & & \\
\hline
\end{tabular}

triggered by a food indiscretion episode, not responsive to the symptomatic therapies employed. The patient's breed and age in this case report, a young West White Highland Terrier female, is in accordance with the demographic profile of patients affected by the disease, where young females are frequently more affected. Additionally, this particular breed is considered predisposed and is well represented in a series of other case reports [19]. Clinical and laboratory changes in patients with hypoadrenocorticism are well known and described in the literature $[12,19,21]$. However, the characterization of the classic laboratory alterations of the disease, and the confirmation of the diagnosis itself, were hampered by the fact that the patient arrived at the Endocrinology Service already receiving prednisolone therapy in a high dose considering the physiological supplementation needed [8,20]. Still, typical changes secondary to hypocortisolism such as hypocholesterolemia, hypotriglyceridemia and hypoproteinemia could be seen in the patient's initial presentation at the referral center [21-23]. In contrast, the high dose of prednisolone used might justify the mature neutrophilia and eosinopenia in the initial blood screening tests, as well as the slightly high blood glucose [20].

Interestingly, the changes resulting from hypoaldosteronism such as hyponatremia and hyperkalemia $[22,23]$ seem to have been partially compensated by the use of a high dose of prednisolone twice a day, associated with oral rehydration with a homemade serum. Notwithstanding, hypochloremia associated with hypoaldosteronism were also demonstrated in the first exams. However, when the dose of prednisolone was reduced and the patient had another gastrointestinal crisis associated with hematoquezia, an important hyponatremia was still observed (Table 1, day 2), even after $12 \mathrm{~h}$ of therapy with $0.9 \% \mathrm{NaCl}$ replacement, oral fludrocortisone, and dexamethasone.

In fact, fludrocortisone is less effective in normalizing electrolytes and plasma renin activity compared to deoxycorticosterone in patients with hypoadrenocorticism [2]. In contrast, the mineralocorticoid action of the latter has the potential to promote hypokalemia, hypernatremia and hypertension in patients receiving an overdose $[2,10]$. Although no serum aldosterone measurement was performed in the present case, the good clinical response and the patient's tolerance to the deoxycorticosterone dose used over time, is compatible with primary hypoadrenocorticism (Addison's syndrome) with aldosterone and cortisol deficiency occurrence in this case [22].

Due to the administration of prednisolone at a dose of $1 \mathrm{mg} / \mathrm{kg}$ the night before the exam, as well as $2 \mathrm{~h}$ before the first blood collection, the classical hormonal documentation of hypoadrenocorticism was impaired. Basal cortisol values above $2 \mu \mathrm{g} / \mathrm{dL}$ rule out the diagnosis of hypoadrenocorticism [3]. However, the baseline cortisol level within the reference range $(4.04 \mu \mathrm{g} / \mathrm{dL})$ probably was secondary to the cross 
reaction between prednisolone and cortisol in the radioimmunoassay used in the test. This is the most probable hypothesis because the post-ACTH cortisol sample result was $50 \%$ lower than the baseline result, reflecting the decline in plasma prednisolone concentration after oral administration. Prednisolone sodium phosphate in oral suspension shows a peak plasma concentration in less than 1 hour after administration, with an elimination rate (t1/2) of about $1 \mu \mathrm{g} / \mathrm{mL} / \mathrm{h}$ [24]. In addition, the fact that the patient received 2 doses of $1 \mathrm{mg} / \mathrm{kg}$ of prednisolone before the ACTH stimulation test was performed does not justify the lack of cortisol response in the test. Suppression of the adrenal hypothalamic-pituitary-cortex axis induced by similar doses of prednisolone is milder and may take weeks to be revealed by ACTH stimulation tests [4,5].

In order to diagnose the primary origin of hypoadrenocorticism, measurement of endogenous ACTH is indicated and necessary to distinguish cases originating from the pituitary gland $[21,22]$. Notwithstanding, the fact that the patient was already receiving high doses of glucocorticoid contraindicated the measurement of endogenous ACTH in the first exams, as the administration of prednisolone, even in low doses (e.g., $0.22 \mathrm{mg} / \mathrm{kg}$ ) causes supression of the hypothalamic-pituitary-adrenalcortex axis [4,5]. Therefore, in the particular case here reported, it could have led to a normal concentration of endogenous ACTH in primary hypoadrenocorticism [20,21]. Thus, the measurement of the patient's endogenous ACTH was performed 6 weeks after the regular administration of $0.15 \mathrm{mg} / \mathrm{kg}$ prednisolone on alternate days. The high concentration of ACTH observed was considered compatible with primary hypoadrenocorticism, since the increase in the eACTH after glucocorticoid therapy in healthy patients is less dramatic [4].

Regarding the probable thalamic ODS documented in this case, is important to notice that myelinolysis/demyelination is an exceedingly rare noninflammatory neurological disorder, initially called central pontine myelinolysis, which can occur after rapid correction of hyponatremia [14]. It has already been documented in dogs after correction of hyponatremia of different origins, including hypoadrenocorticism [16] and parasitic gastrointestinal disorders [7,18]. Currently, the terms "osmotic myelinolysis" or "osmotic demyelination syndrome" are considered more suitable when compared to the term "central pontine myelinolysis" since it has been demonstrated in dogs and humans the occurrence of demyelination secondary to the rapid correction of hyponatremia in distinct regions of the central nervous system including pons, basal nuclei, striatum, thalamus, cortex, hippocampus, and cerebellum $[9,18]$.

The complex mechanisms behind the induction of osmotic myelinolysis syndrome after a rapid rise in sodium concentration still need more studies $[6,16]$, despite advances have been made in understanding the susceptibility of glial cells (oligodendrocytes and astrocytes) to this phenomenon [17]. Histologically, the destruction of the myelin sheath leading to scarcity of neuronal bodies and axons can be observed, whereas neuroinflammation only occurs after a well-established myelinolysis [7,9]. Experimental studies [15] and case reports in dogs [18] and humans [9] have shown that there may be a difference between the emergence of clinical signs and the evidence of myelinolysis on MRI images. Regarding the lesions itself, a sequential evolution intensity, severity and areas of the central nervous system affected can also be observed. In addition, clinical signs often vary in severity from mild disorientation to death, occurring two to 7 days after exposure to a rapid increase in plasma sodium [15].

In the present case report, the probable moment of myelinolysis induction occurred on the reported second day. At that time, since the owners did not authorize the initial admission to the Endocrinology Service in order to stabilize the patient, a reduction in the dose of prednisolone associated with the still recent introduction of fludrocortisone was performed. Apparently, the patient did not tolerate this therapeutic adjustment, which caused a severe hemorrhagic gastroenteritis crisis, leading to an emergency admission to a private clinic. Although the rate of sodium chloride administered during hospitalization was not made available to the owners, blood tests were performed only $12 \mathrm{~h}$ after the beginning of the therapy on day 2 . It still showed a low serum sodium concentration of $130 \mathrm{mmol} / \mathrm{L}$, which was already within the reference range in the next day (data not shown), due to the maintenance of fluid therapy. After 5 days of this episode, the patient started to present neurological clinical signs.

Despite MRI was performed only 7 days after the initial visit at the neurology service (day 7) due to owners' private issues, extremely symmetrical changes in hypersignal in T2-weighted and Flair images on MRI 
bilaterally in the central thalamic region were considered compatible with the later aspect of MRI osmotic myelinolysis, as demonstrated in a previous study [15]. The absence of changes in CSF analysis (Table 2) also supports the hypothesis of demyelination since it is an essentially non-inflammatory disorder $[9,14]$. Interestingly, no specific management for myelinolysis was performed in this case, other than keeping hypoadrenocorticism therapy. The potential for mortality or permanent neurological sequelae after osmotic myelinolysis is described in the literature $[9,16]$. However, the early administration of glucocorticoids in human patients with osmotic myelinolysis has been proposed as a key factor for rapid recovery and absence of sequelae from this syndrome [1]. Thus, it is possible that in the present case, the favorable outcome was due to the administration of dexamethasone for initial glucocorticoid supplementation at admission, associated with the prednisolone maintenance along with fludrocortisone in the first days after the neurological crisis. A similarly favorable outcome was previously described in a case report with neurological complications secondary to hypoadrenocorticism treatment [16].

The findings here described reveal important questions regarding the diagnosis and management of hypoadrenocorticism. First, the importance of hormonal confirmation of the diagnosis through ACTH stimulation tests and measurement of eACTH, associated with the assessment of mineralocorticoid function through the evaluation of serum electrolytes, which should be performed before the beginning of hydroelectrolytic replacement therapy and supplementation of glucocorticoids and mineralocorticoids [13,21]. Also, the treatment of hypoadrenocorticism should focus on correcting hypotension, hypovolemia, and electrolyte alterations; while promoting an adequate supplementation of glucocorticoids and mineralocorticoids [16,21] avoiding excessive electrolyte and corticoid supplementation in order to prevent osmotic myelinolysis, Cushing syndrome and/or Conn syndrome. Finally, the standard therapy of glucocorticoid supplementation in hypoadrenocorticism may be enough to support the neurological recovery of patients with ODS, unlike cases of osmotic myelinolysis induced by other causes of hyponatremia, which may require administration of glucocorticoids in therapeutic doses to reduce the syndrome morbidity and mortality [1].

\section{MANUFACTURERS}

${ }^{1}$ Cosmed Indústria de Cosméticos e Medicamentos S.A. Barueri, SP, Brazil.

${ }^{2}$ Biolab Sanus Farmacêutica Ltda. Taboão da Serra, SP, Brazil.

${ }^{3}$ Medley Farmacêutica Ltda. Campinas, SP, Brazil.

${ }^{4}$ Mallinckrodt Pharmaceuticals plc. Staines-upon-Thames, Surrey, UK.

${ }^{5}$ MP Biomedicals. Solon, OH, USA.

${ }^{6}$ Esaote SpA. Firenze, Tuscany, Italy.

${ }^{7}$ Bristol-Myers Squibb. New York, NY, USA.

${ }^{8}$ Merck Sharp \& Dohme Saúde Animal Ltda. São Paulo, SP, Brazil.

${ }^{9}$ Dechra Pharmaceuticals PLC. Northwich, Cheshire, UK.

${ }^{10}$ Siemens AG. Munich, Bavaria, Germany.

Acknowledgements. The authors would like to thank VMD, Ph.D., DACVIM Ellen Behrend and BVSc, MVetClinStud, FANZCVS, Dr. Sue Foster for their help with the ACTH stimulation test and eACTH concentration test design and interpretation.

Declaration of interest. The authors report no conflicts of interest. The authors alone are responsible for the content and writing of the paper.

\section{REFERENCES}

1 Bansal L.R. 2018. Therapeutic effect of steroids in osmotic demyelination of infancy. Child Neurology Open. 5: 1-4.

2 Baumstark M.E., Sieber-Ruckstuhl N.S., Müller C., Wenger M., Boretti F.S. \& Reusch C.E. 2014. Evaluation of aldosterone concentrations in dogs with hypoadrenocorticism. Journal of Veterinary Internal Medicine. 28(1): 154-159.

3 Bovens C., Tennant K., Reeve J. \& Murphy K.F. 2014. Basal serum cortisol concentration as a screening test for hypoadrenocorticism in dogs. Journal of Veterinary Internal Medicine. 28(6): 1541-1545.

4 Brockus C.W., Dillon A.R. \& Kemppainen R.J. 1999. Effect of alternative-day prednisolone administration on hypophyseal-adrenocortical activity in dogs. American Journal of Veterinary Research. 60(6): 698-702.

5 Chastain C.B. \& Graham C.L. 1979. Adrenocortical suppression in dogs on daily and alternate-day prednisone administration. American Journal of Veterinary Research. 40(7): 936-941.

6 Church D.B. 2012. Canine hypoadrenocorticism. In: Mooney C.T. \& Peterson M.E. (Eds). BSAVA Manual of Canine and Feline Endocrinology. 4th edn. Gloucester: BSAVA, pp.156-166.

7 Churcher R.K., Watson A.D.J. \& Eaton A. 1999. Suspected myelinolysis following rapid correction of hyponatremia in a dog. Journal of the American Animal Hospital Association. 35(6): 493-497. 
8 Farr H., Mason B.L. \& Longhofer S.L. 2020. Randomised clinical non-inferiority trial comparing two formulations of desoxycortone pivalate for the treatment of canine primary hypoadrenocorticism. Veterinary Record. 187(2): e12.

9 Garg P., Aggarwal A., Malhotra R. \& Dhall S. 2019. Osmotic demyelination syndrome - Evolution of extrapontine before pontine myelinolysis on magnetic resonance imaging. Journal of Neurosciences in Rural Practice. 10(1): 126135.

10 Jaffey J.A., Nurre P., Cannon A.B. \& Declue A.E. 2017. Desoxycorticosterone pivalate duration of action and individualized dosing intervals in dogs with primary hypoadrenocorticism. Journal of Veterinary Internal Medicine. 31(6): 1649-1657.

11 Lathan P. 2013. Hypoadrenocorticism in dogs. In: Rand J. (Ed). Clinical Endocrinology of Companion Animals. Ames: Wiley-Blackwell, pp.1-21.

12 Lathan P. \& Tyler J. 2005. Canine hypoadrenocorticism: diagnosis and treatment. Compendium of Continuous Education in Veterinary. 27(2): 121-131.

13 Lathan P. \& Tyler J. 2005. Canine hypoadrenocorticism: pathogenesis and clinical features. Compendium of Continuous Education in Veterinary. 27(2): 110-120.

14 Laureno R. \& Karp B.I. 1997. Myelinolysis after correction of hyponatremia. Annals of Internal Medicine. 126(1): 57-62.

15 Laureno R., Lamotte G. \& Mark A.S. 2018. Sequential MRI in pontine and extrapontine myelinolysis following rapid correction of hyponatremia. BMC Research Notes. 11(1): 707-711.

16 MacMillan K.L. 2003. Neurologic complications following treatment of canine hypoadrenocorticism. Canadian Veterinary Journal. 44(6): 490-492.

17 Nicaise C., Marneffe C., Bouchat J. \& Gilloteaux J. 2019. Osmotic demyelination: from an oligodentrocyte to an astrocyte perspective. International Journal of Molecular Sciences. 20(5): 1124-1141.

18 O'Brien D.P., Kroll R.A., Johnson G.C., Covert S.J. \& Nelson M.J. 1994. Myelinolysis after correction of hyponatremia in two dogs. Journal of Veterinary Internal Medicine. 8(1): 40-48.

19 Peterson M.E., Kintzer P.P. \& Kass P.H. 1996. Pretreatment clinical and laboratory findings in dogs with hypoadrenocorticism: 225 cases (1979-1993). Journal of the American Veterinary Medical Association. 208(1): 85-91.

20 Reusch C.E. 2015. Glucocorticoid Therapy. In: Feldman E.C. \& Nelson R.W. (Eds). Canine \& Feline Endocrinology. 4th edn. St. Louis: Elsevier Saunders, pp.555-577.

21 Scott-Moncrieff J.C. 2015. Hypoadrenocorticism. In: Feldman E.C. \& Nelson R.W. (Eds). Canine \& Feline Endocrinology. 4th edn. St. Louis: Elsevier Saunders, pp.458-520.

22 Shiel R.E. \& Mooney C.T. 2019. Redefining the paradigm of atypical hypoadrenocorticism in dogs. Companion Animal. 24(2): 14-22.

23 Thompson A.L., Scott-Moncrieff J.C. \& Anderson J.D. 2007. Comparison of classic hypoadrenocorticism with glucocorticoid-deficient hypoadrenocorticism in dogs: 46 cases (1985-2005). Journal of the American Veterinary Medical Association. 230(8): 1190-1194.

24 Tse F.L. \& Welling P.G. 1977. Prednisolone bioavailability in the dog. Journal of Pharmaceutical Sciences. 66(12): 1751-1754. 